\title{
Nondestructive Imaging of Plant-Parasitic Nematode Development and Host Response to Nematode Pathogenesis
}

\author{
Phuong T. Y. Dinh, Michael Knoblauch, and Axel A. Elling
}

First and third authors: Department of Plant Pathology, and second author: School of Biological Sciences, Washington State University, Pullman 99164.

Accepted for publication 21 November 2013.

\section{ABSTRACT}

Dinh, P. T. Y., Knoblauch, M., and Elling, A. A. 2014. Nondestructive imaging of plant-parasitic nematode development and host response to nematode pathogenesis. Phytopathology 104:497-506.

The secluded lifestyle of endoparasitic plant nematodes hampers pro-
gress toward a comprehensive understanding of plant-nematode inter-
actions. A novel technique that enables nondestructive, long-term obser-
vations of a wide range of live nematodes in planta is presented here. As
proof of principle, Pratylenchus penetrans, Heterodera schachtii, and
Meloidogyne chitwoodi were labeled fluorescently with PKH26 and used
to infect Arabidopsis thaliana grown in microscopy rhizosphere cham-
bers. Nematode behavior, development, and morphology were observed
for the full duration of each parasite's life cycle by confocal microscopy for up to 27 days after inoculation. PKH26 accumulated in intestinal lipid droplets and had no negative effect on nematode infectivity. This technique enabled visualization of Meloidogyne gall formation, nematode oogenesis, and nematode morphological features, such as the metacorpus, vulva, spicules, and cuticle. Additionally, microscopy rhizosphere chambers were used to characterize plant organelle dynamics during $M$. chitwoodi infection. Peroxisome abundance strongly increased in early giant cells but showed a marked decrease at later stages of feeding site development, which suggests a modulation of plant peroxisomes by root-knot nematodes during the infection process. Taken together, this technique facilitates studies aimed at deciphering plant-nematode interactions at the cellular and subcellular level and enables unprecedented insights into nematode behavior in planta.
Plant-parasitic nematodes are a major threat to sustainable crop production in temperate and tropical regions alike. Some of the most damaging species belong to the root-lesion (Pratylenchus spp.), cyst (Globodera and Heterodera spp.), and root-knot (Meloidogyne spp.) nematodes $(11,22)$. All plant-parasitic nematodes are obligate biotrophs that depend on their hosts for survival. However, infection strategies can show marked differences between genera. Root-lesion nematodes are migratory endoparasites that invade roots and feed on a large number of host cells that are destroyed in the process, thereby resulting in lesions on the roots (54). In contrast, sedentary endoparasites such as cyst and root-knot nematodes induce the formation of living feeding sites in infected plant tissue and minimize cellular damage to their hosts. Syncytia and giant cells, the feeding sites of cyst and rootknot nematodes, respectively, are the sole source of nutrition for these nematodes and, therefore, essential to their survival (26). The basic principles of the life cycles of root-lesion, cyst, and root-knot nematodes are relatively similar. All four juvenile life stages (J1, J2, J3, and J4) and the development into either adult males or females are separated by molts. The first-stage juvenile (J1) remains in the egg and molts into a second-stage juvenile (J2), which hatches. In cyst and root-knot nematodes, the $\mathbf{J} 2$ is the infective stage that invades plant tissue and initiates the formation of a feeding site, whereas the remaining life stages (except the adult male) are sedentary (26). In root-lesion nematodes, all life stages from the J2 onward are motile and infective (54). An important difference in postinfection biology is that, in Meloidogyne

Corresponding author: A.A. Elling; E-mail address: elling@wsu.edu

* The $e$-Xtra logo stands for "electronic extra" and indicates that Figures 1 to 5 appear in color online.

http://dx.doi.org/10.1094/PHYTO-08-13-0240-R

(c) 2014 The American Phytopathological Society spp., J3 and J4 stages do not feed because they remain ensheathed in the cuticles of the previous life stage and lose their stylets. In contrast, in Heterodera spp., the cuticle of the previous life stage is completely shed and the J3 and J4 nematodes feed. In Pratylenchus spp., all vermiform life stages feed (37).

The ability to study the behavior of plant-parasitic nematodes during pathogenesis and to characterize the cellular changes in infected plant tissue is of great interest, not only to elucidate fundamental principles of host-parasite interactions but also to explore novel control strategies. Plant-parasitic nematodes secrete proteinaceous effectors from the esophageal gland cells into plant tissue to induce cellular and physiological changes in the host (33) but the basic mechanisms that define molecular and cellular plant-nematode interactions remain largely unknown. One of the reasons that significant advances in this area are slow is that endoparasitic nematodes are inherently difficult to study. As obligate biotrophs, they cannot be propagated without their hosts and, once they are deeply embedded in plant tissue, key events become obscured. One of the most commonly used methods to analyze plant-nematode relationships relies on fixing infected roots and cross-sectioning areas of interest. Depending on the research question of interest, cross-sectioning is often combined with histological or immunological staining and subsequent observations by light or fluorescence microscopy $(2,38,47,48)$ or followed up with electron microscopy $(14,19,23,24,44)$. Even though this approach has yielded valuable insights, it has shortcomings that hamper conceptual breakthroughs. Cross-sectioning plant and nematode tissue is technically demanding, destructive, and prone to fixation artifacts. Furthermore, the results give static snapshots, and do not provide a dynamic picture of cellular changes or nematode behavior over time. Whole-mounts are an alternative to cross-sections and facilitate sample preparation but are subject to the same limitations $(16,49)$. To partially overcome these problems, previous investigators have used video light microscopy (43) or fluorescent dyes to track nematode infection $(15,42)$. However, in these cases, 
either the dye was lost by the nematode after only a few days or the experimental setup did not allow for in-depth in planta studies. Furthermore, most approaches are based on artificial growing conditions (i.e., plants maintained in agar or as root explants, methods that can substantially alter plant-nematode interactions).

Here, we report on an optimized technique that overcomes these challenges by fluorescently labeling root-lesion nematodes (Pratylenchus penetrans), sugar beet cyst nematodes (Heterodera schachtii), and Columbia root-knot nematodes (Meloidogyne chitwoodi) with the lipid analog PKH26 and, subsequently, following the behavior of labeled nematodes in Arabidopsis thaliana grown in microscopy rhizosphere chambers (micro-ROC) (12) using confocal microscopy throughout each life cycle. PKH26 is a lipophilic fluorescent dye with long aliphatic tails that integrates into lipids and is widely used for long-term cell tracking assays in mammalian systems (41). PKH26 has been used to study the surface coat of nematodes $(32,34,35)$ but its potential to observe live nematodes over longer periods in planta has not been examined to date.

\section{MATERIALS AND METHODS}

Nematode inoculum. Nematode stock cultures were maintained under greenhouse conditions. As host plants, Mentha $\times$ piperita 'Black Mitcham' was used for P. penetrans, Beta vulgaris '4430R' (Betaseed, Shakopee, MN) for H. schachtii, and Solanum lycopersicum 'Rutgers' for Meloidogyne chitwoodi. All host plants were grown in autoclaved sand. To obtain inoculum for experiments, mixed life stages of $P$. penetrans were extracted by cutting infected mint roots into $1-$ to $2-\mathrm{cm}$ pieces and shaking them in a dilute $\mathrm{NaOCl}$ solution $(0.01 \%$ commercial bleach) on a rotary shaker at $200 \mathrm{rpm}$ for 2 days at room temperature $\left(23^{\circ} \mathrm{C}\right)$. The suspension was poured through a set of nested sieves (850-, $75-$, and $25-\mu \mathrm{m}$ pore size from top to bottom). Each sieve was consecutively rinsed with water for 2 min. Mixed life stages of $P$. penetrans collected on the $25-\mu \mathrm{m}$ pore sieve were backwashed into a 50-ml tube and purified on a sucrose gradient following established procedures (17) before being used for subsequent experiments. $H$. schachtii cysts were collected by mixing heavily infested sand from sugar beet stock cultures with water and decanting the supernatant over a $250-\mu \mathrm{m}$ pore sieve. Cysts were

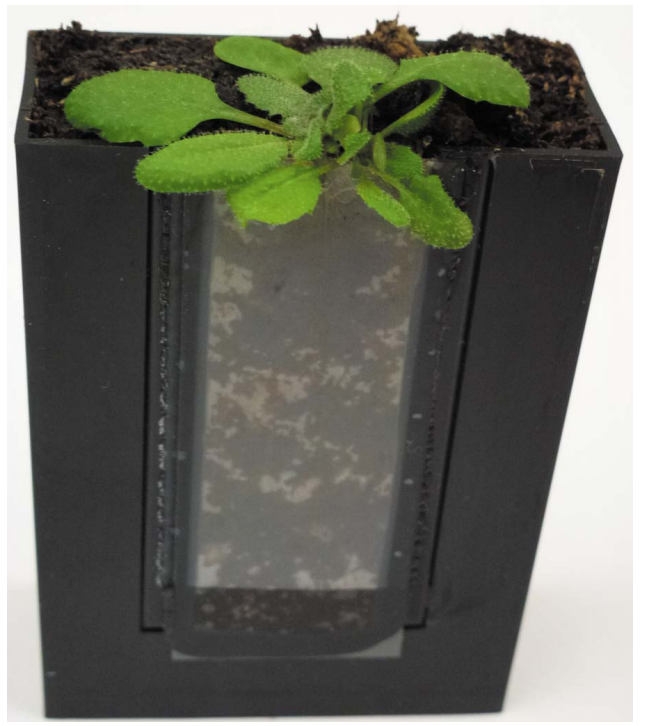

Fig. 1. Arabidopsis grown in microscopy rhizosphere chambers (micro-ROC). For all experiments, a single Arabidopsis seedling was grown in each microROC. The developing root system grows between a nylon mesh and glass slide but has access to the potting mix. The glass slide is covered with a black plastic film (removed here) to block out light. Nematode inoculum was pipetted between the nylon mesh and glass slide to maximize infection. rinsed with water and crushed by manually rubbing them against the sieve surface, and the released eggs were collected on a $25-\mu \mathrm{m}$ pore sieve. $H$. schachtii eggs were purified on a sucrose gradient (17) and incubated in a modified Baermann pan at room temperature $\left(23^{\circ} \mathrm{C}\right)$ to allow hatching of $\mathrm{J} 2 \mathrm{~s}$. The hatch solution contained $3.14 \mathrm{mM} \mathrm{ZnSO}_{4}$ at $\mathrm{pH}$ 7.0. Then, 4 to 7 days later, J2s were collected by centrifuging the hatch suspension for $3 \mathrm{~min}$ at $375 \times g$ in a HN-S clinical centrifuge (International Equipment Co., Needham Heights, MA). The resulting nematode pellet was washed with sterile water and centrifuged once more before being used for PKH26 labeling. To obtain M. chitwoodi inoculum, eggs were extracted from infested tomato roots using $0.5 \% \mathrm{NaOCl}$ (18), then purified on a sucrose gradient (17). Eggs were rinsed with water and transferred to a modified Baermann pan to hatch $\mathrm{J} 2 \mathrm{~s}$ in sterile water at room temperature $\left(23^{\circ} \mathrm{C}\right)$. After 4 days, $\mathrm{J} 2 \mathrm{~s}$ were collected by centrifuging the hatch suspension, as described above. Nematodes were washed with sterile water and centrifuged once more before being used for PKH26 labeling.

PKH26 labeling. In total, $\approx 50,000$ mixed life stages of $P$. penetrans and $\mathrm{J} 2 \mathrm{~s}$ of $H$. schachtii and $M$. chitwoodi were transferred to separate $1.5-\mathrm{ml}$ microcentrifuge tubes and resuspended in $1 \mathrm{ml}$ of sterile water before $1 \mu \mathrm{l}$ of PKH26 (stock solution $1 \times 10^{-3} \mathrm{M}$ ) from the MINI26 PKH26 Red Fluorescent Cell Linker Kit (Sigma-Aldrich, St. Louis, MO) was added. Each tube was inverted several times to mix the nematode suspensions, then incubated in the dark at room temperature for $15 \mathrm{~min}$. To remove excess dye, nematodes were washed five times by repeated transfers to $50 \mathrm{ml}$ of sterile water followed by centrifuging at $375 \times g$ for $3 \mathrm{~min}$ in a clinical centrifuge as described above. Stained nematodes were immediately used to inoculate Arabidopsis. One aliquot of each batch of PKH26-labeled inoculum was stored in water at $4{ }^{\circ} \mathrm{C}$ to observe fluorescence of stained nematodes outside the host plant.

Micro-ROC. A. thaliana ecotype Col-0 seed were incubated in sterile water at $4^{\circ} \mathrm{C}$ for 2 days, after which seed were planted individually in micro-ROC (Advanced Science Tools, Pullman, WA) containing moistened Sunshine number 1 potting mix (Sun Gro Horticulture, Agawam, MA) (Fig. 1). Plants were grown in a growth room with a 14-h photoperiod (300 to $400 \mathrm{mEm}^{-2} \mathrm{~s}^{-1}$ ) and a day-and-night temperature cycle of 20 and $15^{\circ} \mathrm{C}$, respectively. Ten days after planting, when the four-leaf stage was reached, each seedling was inoculated with $\approx 2,000$ stained nematodes by pipetting $100 \mu \mathrm{l}$ of nematode suspension between the glass slide and the nylon mesh of each micro-ROC. Beginning at 3 days after inoculation (DAI), plants were carefully watered and fertilized with modified Knop's media (43) without sucrose. For each nematode species, 15 micro-ROC containing one A. thaliana seedling each were inoculated. As controls, uninfected plants and unlabeled nematodes were used. All experiments were conducted twice.

Arabidopsis px-YFP line. Binary plasmid px-YFP (TAIR accession CD3-982) (36) was introduced into Agrobacterium tumefaciens strain GV3101 and used to transform Arabidopsis thaliana ecotype Col-0 using the floral dip method (7). This created an Arabidopsis line in which all peroxisomes were labeled fluorescently by translationally fusing the peroxisomal targeting signal 1 (PTS1, Ser-Lys-Leu) to the C terminus of the yellow fluorescent protein (YFP). Transformants were selected by screening germinating seed on one-half strength Murashige \& Skoog basal salt media (Caisson Labs, North Logan, UT), supplemented with 3\% D-sucrose, $0.6 \%$ Daishin agar, timentin at $0.5 \mathrm{~g} / \mathrm{liter}$, and glufosinate ammonium (Plant Media, Dublin, $\mathrm{OH}$ ) at $10 \mu \mathrm{g} / \mathrm{ml}$. Second-generation $\left(\mathrm{T}_{2}\right)$ seed were used in micro-ROC experiments as described above, except that plants were inoculated with unlabeled M. chitwoodi. Fifteen micro-ROC with one plant each were analyzed and the experiment was conducted twice. Wildtype Arabidopsis and uninfected px-YFP plants were used as controls. For each time point $(3,8,12,19$, and $27 \mathrm{DAI})$, the num- 
ber of peroxisomes was counted in three areas of three images each in infected and uninfected roots following previously established procedures (9). Briefly, three $0.01-\mathrm{mm}^{2}$ areas (100 by $100 \mu \mathrm{m})$ were cropped from each image using Adobe Photoshop. Using ImageJ, cropped RGB color images were changed to 8-bit black-and-white images. The "threshold" function was used to designate black pixels as peroxisome area. The "analyze particles" function was used to count pixel groups (number of peroxisomes) and area fraction (area percentage). This technique was unable to differentiate between overlapping peroxisomes, which were counted as one organelle and possibly reduced the true number of peroxisomes in some instances. To circumvent this problem, the total area of peroxisomes was estimated ([area fraction/100] $\times$ $0.01 \mathrm{~mm}^{2}$ ). All data were analyzed for statistical significance using a $t$ test at $\alpha 0.05$ using SAS 9.2 software.

Confocal microscopy. Infected Arabidopsis roots and uninfected controls grown in micro-ROC were observed using an LSM 510 META laser scanning microscope (Zeiss, Jena, Germany) beginning at 3 DAI and extending for up to 27 DAI. Excitation wavelengths were set at $514 \mathrm{~nm}$ (argon) and $543 \mathrm{~nm}$ $(\mathrm{HeNe})$ with emission wavelengths of 503 to $530 \mathrm{~nm}$ (green) and
560 to $615 \mathrm{~nm}$ (red). In addition, nematodes stained with PKH26 were observed on standard microscopy glass slides 3 days after being labeled using the same parameters. For px-YFP roots and respective controls, the excitation wavelengths were set at $514 \mathrm{~nm}$ (argon) with the emission wavelength at $560 \mathrm{~nm}$. Subsequent image processing was performed with ImageJ and Adobe Photoshop.

\section{RESULTS}

P. penetrans behavior and life cycle in Arabidopsis roots. Immediately after being labeled with $\mathrm{PKH} 26$, all vermiform life stages of $P$. penetrans began to show fluorescence in the lip region, esophagus, and intestine. At 3 days after labeling, pronounced staining was visible, especially in lipid droplets in the intestine of $P$. penetrans that had been stored at $4^{\circ} \mathrm{C}$ (Fig. 2A). Increased mortality of labeled $P$. penetrans or overtly toxic effects of PKH26 compared with untreated nematodes were not observed. To examine whether micro-ROC facilitates observation of root-lesion nematode behavior in planta and throughout the life cycle of the parasite, Arabidopsis grown in micro-ROC were inoculated with mixed life stages of $P$. penetrans that were labeled
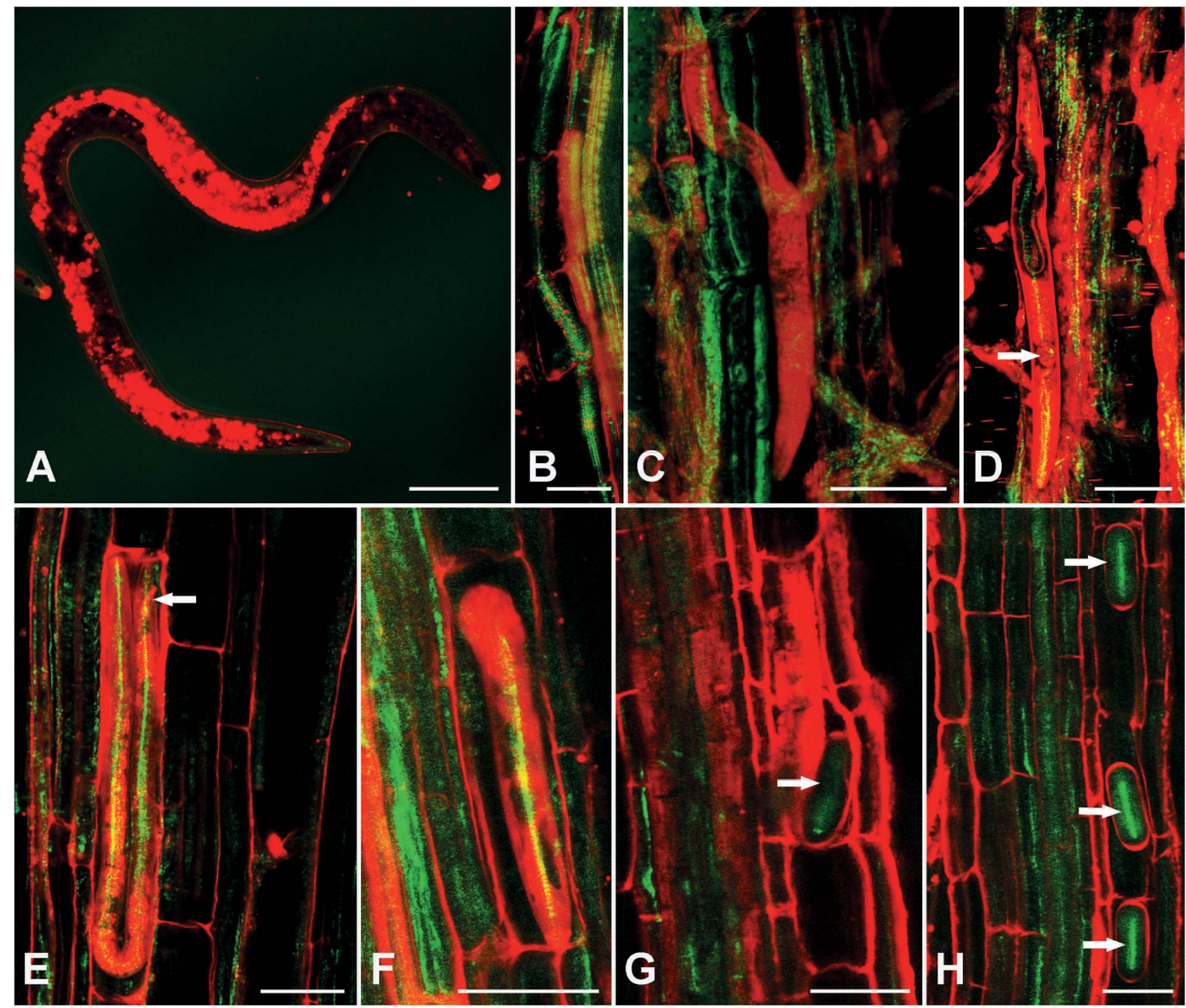

Fig. 2. Pratylenchus penetrans stained with $\mathrm{PKH} 26$. A, P. penetrans 3 days after labeling with $\mathrm{PKH} 26$ outside of plant, stored at $4{ }^{\circ} \mathrm{C}$ until imaging. Intense fluorescence of intestinal lipids. All other pictures show nematodes in planta. B, P. penetrans at 3 days after inoculation (DAI) in Arabidopsis roots and $\mathbf{C}$, at 19 DAI. D, Molting female at 19 DAI; arrow indicates metacorpus. E, Resting male at 26 DAI, arrow indicates spicules. F, Anterior portion of female during migration through epidermal tissue at 27 DAI. G, Migrating female at 27 DAI with deposited egg (arrow). H, Deposited eggs at 27 DAI (arrows). Scale bars = $50 \mu \mathrm{m}$. 
with PKH26. The nematodes migrated toward and invaded the roots as early as 1 DAI. At 3 DAI, the fluorescence of lipid droplets temporarily decreased in $\mathrm{PKH} 26$-stained $P$. penetrans inside roots but rapidly recovered in feeding nematodes (Fig. 2B and C). Nematodes were observed for up to 27 DAI, at which point $P$. penetrans completed its life cycle. The intensity of PKH26 fluorescence was strong throughout the duration of the experiments and did not weaken at 27 DAI. Importantly, microROC enabled observation of characteristic $P$. penetrans behavior throughout the nematode's life cycle, including intracellular migration (Fig. 2B and C), feeding (Fig. 2C and D), coiling and resting inside host cells (Fig. 2E and F), and deposition of eggs inside host tissue (Fig. 2G and H). In addition, distinct morphological features such as the intestine, metacorpus, vulva (Fig. 2D), and spicules (Fig. 2E) were visible in labeled nematodes inside roots.

H. schachtii behavior and life cycle in Arabidopsis roots. After PKH26 and micro-ROC were used to observe the migratory endoparasite $P$. penetrans in planta, the technique was tested in sedentary endoparasitic nematodes. $H$. schachtii infective J2s were labeled and used to inoculate Arabidopsis grown in microROC, as detailed above. Similar to $P$. penetrans, $H$. schachtii infective J2s began to show PKH26-induced fluorescence immediately after being exposed to the dye. At 3 days after labeling, intestinal lipid droplets and the lip region displayed strong fluorescence in labeled $\mathrm{J} 2 \mathrm{~s}$ that were stored at $4^{\circ} \mathrm{C}$ (Fig. 3A). PKH26 did not have any obvious effects on the viability of $\mathrm{J} 2 \mathrm{~s}$, which oriented themselves toward host roots, explored, and finally invaded roots at the tips and in the region of cell differentiation. The fluorescence of lipid droplets temporarily decreased in nonfeeding infective J2s at 10 DAI (Fig. 3B) but quickly increased in feeding $\mathrm{J} 3 \mathrm{~s}$ at $14 \mathrm{DAI}$ and even surpassed the staining intensity of $\mathrm{J} 2 \mathrm{~s}$ in later life stages (late $\mathrm{J} 3$ and $\mathrm{J} 4$ ) at 22 DAI and beyond (Fig. 3C to G). In developing females, fluorescent lipid droplets decreased and almost disappeared in adult females and cysts (Fig. $3 \mathrm{H}$ and I). Although lipid staining decreased in adult females and cysts, residual PKH26 staining of the remaining lipid droplets, together with autofluorescence in the outer layers of the cyst, enabled a clear visualization of these important life stages. Combined with PKH26, micro-ROC enabled observation of all phases of the $H$. schachtii life cycle in great detail in planta, including migration through host tissue (Fig. 3B), feeding (Fig. 3C and D), molting (Fig. 3E), development of vermiform males and female cysts (Fig. 3G to I), oogenesis (Fig. 3H and I), and formation of the syncytium (Fig. 3J). Furthermore, morphological details of live $H$. schachtii were clearly visible in planta, and included the esophagus, metacorpus (Fig. 3D), intestine, (Fig. 3D to G), shed cuticle during molt (Fig. 3E), ovaries (Fig. 3H), and vulval cone (Fig. 3I).

M. chitwoodi behavior and life cycle in Arabidopsis roots. Similar to the other nematode species studied, $M$. chitwoodi infective J2s began to display fluorescence immediately upon exposure to PKH26. At 3 days after labeling, the lip region and lipid droplets in the intestine of $\mathrm{J} 2 \mathrm{~s}$ that were stored at $4{ }^{\circ} \mathrm{C}$ showed a strong fluorescent signal (Fig. 4A). As in P. penetrans and H. schachtii, PKH26 did not have any obvious effects on the ability of $M$. chitwoodi infective $\mathrm{J} 2 \mathrm{~s}$ to locate and penetrate host roots. The labeled J2s migrated toward the tips of Arabidopsis roots and invaded roots at the zone of cell elongation. At 3 DAI, stained J2s could be clearly observed during their intercellular migration in root tissue. The fluorescence intensity of their intestinal lipid droplets was high at $3 \mathrm{DAI}$; decreased by the time they had become parasitic $\mathrm{J} 2 \mathrm{~s}$, when they began to induce the formation of feeding sites (Fig. 4B to F); and returned to strong intensity in late feeding life stages at 15 DAI (Fig. 4G). In contrast to developing Heterodera nematodes, which break through the root cortex, especially in roots with a small diameter such as Arabidopsis and thereby facilitate imaging, Meloidogyne nematodes remain deeply em- bedded in root tissue and are surrounded by a gall. As expected, gall formation made it challenging to clearly visualize morphological details in adult $M$. chitwoodi females at 27 DAI, when the life cycle was complete with commencement of egg laying and observations ended (Fig. 4H and I). However, PKH26 stained the cell membranes of giant cells, which greatly facilitated nondestructive observation of feeding site morphology and development and showed the increase in giant cell size compared with infected plant cells (Fig. 4D and F).

M. chitwoodi infection modulates peroxisome abundance in giant cells in Arabidopsis. To test whether the micro-ROC technique is useful for analyzing subcellular changes in nematodeinfected plant tissue, a transgenic Arabidopsis line (px-YFP) in which the peroxisomal targeting signal 1 (PTS1, Ser-Lys-Leu) is fused to the $\mathrm{C}$ terminus of YFP and, thereby, fluorescently labeling peroxisomes (36), was inoculated with $M$. chitwoodi infective J2s. Root tissue peroxisomes were evenly distributed throughout uninfected roots (Fig. 5). At 3 DAI, M. chitwoodi initiated feeding site formation and the number and area of peroxisomes per cell significantly increased in infected root cells (Figs. 5 and 6). At $8 \mathrm{DAI}$, the number of peroxisomes was higher in infected plant cells when observed with the microscope (Fig. 5). However, due to technical constraints that did not allow differentiating single peroxisomes that overlapped in the microscope image, the counting technique used indicated that there was no statistically significant difference in the number of peroxisomes between infected and uninfected cells at 8 DAI (Fig. 6A). Measuring the area of peroxisomes was not affected by these technical constraints (see Materials and Methods) and showed a significant increase in the area of peroxisomes in infected cells at 8 DAI (Fig. 6B). At later stages of the infection cycle, the relative distribution of peroxisomes reversed. At $12 \mathrm{DAI}$, there were significantly fewer peroxisomes in giant cells than in the surrounding plant cells and uninfected areas. This trend continued throughout the remaining time points at 19 and 27 DAI, when the life cycle of $M$. chitwoodi was complete and observations ended (Fig. 5D to G). Taken together, we found that the number of peroxisomes increased in young giant cells compared with uninfected plant tissue whereas, in later stages of the $M$. chitwoodi life cycle, there were significantly fewer peroxisomes in nematode feeding sites relative to in neighboring uninfected plant tissue.

\section{DISCUSSION}

Attempts to study live nematodes in their hosts go back to the early 1940s with the pioneering experiments of Linford, who developed root observation boxes to observe Meloidogyne spp. $(27,28)$. Even though this method allowed root development in soil, thereby having the advantage of offering relatively natural growing conditions, Linford's design did not enable prolonged in planta studies. Early in planta nematode behavior could be imaged inside the root observation boxes but nematode migration events and later behavior could only be followed in dissected roots, which limited the usefulness of this technique (28). Significant breakthroughs in plant-nematode imaging were made by growing Arabidopsis in agar under axenic conditions, infecting plants with surface-sterilized nematodes, and observing the resulting interactions with video-enhanced contrast light microscopy $(14,43,50,51)$. A major advantage of this approach was that it was nondestructive and enabled long-term observations of live nematodes in planta. Subsequent studies made use of fluorescent dyes or plant reporter genes to visualize details of live plantnematode interactions $(6,45)$. However, even minor changes in the composition of artificial plant growth media can have a major impact on the infection rate of nematodes and, therefore, alter the relationships with the host, which creates a major challenge when using axenic growth conditions (43). Furthermore, imaging of live nematodes during the infection process would greatly benefit 
from a fluorescent label that could be applied to the parasite and facilitate following its behavior in planta. Plant-parasitic nematodes are known to take up fluorescent dyes such as acridine orange, fluorescein isothiocyanate, or fluorescein diacetate $(4,5$,
15,42). However, these dyes proved to be of relatively little value in plant-nematode interaction studies because they were not stable throughout the entire life of the nematode, thereby obscuring late parasitic events $(15,42)$.

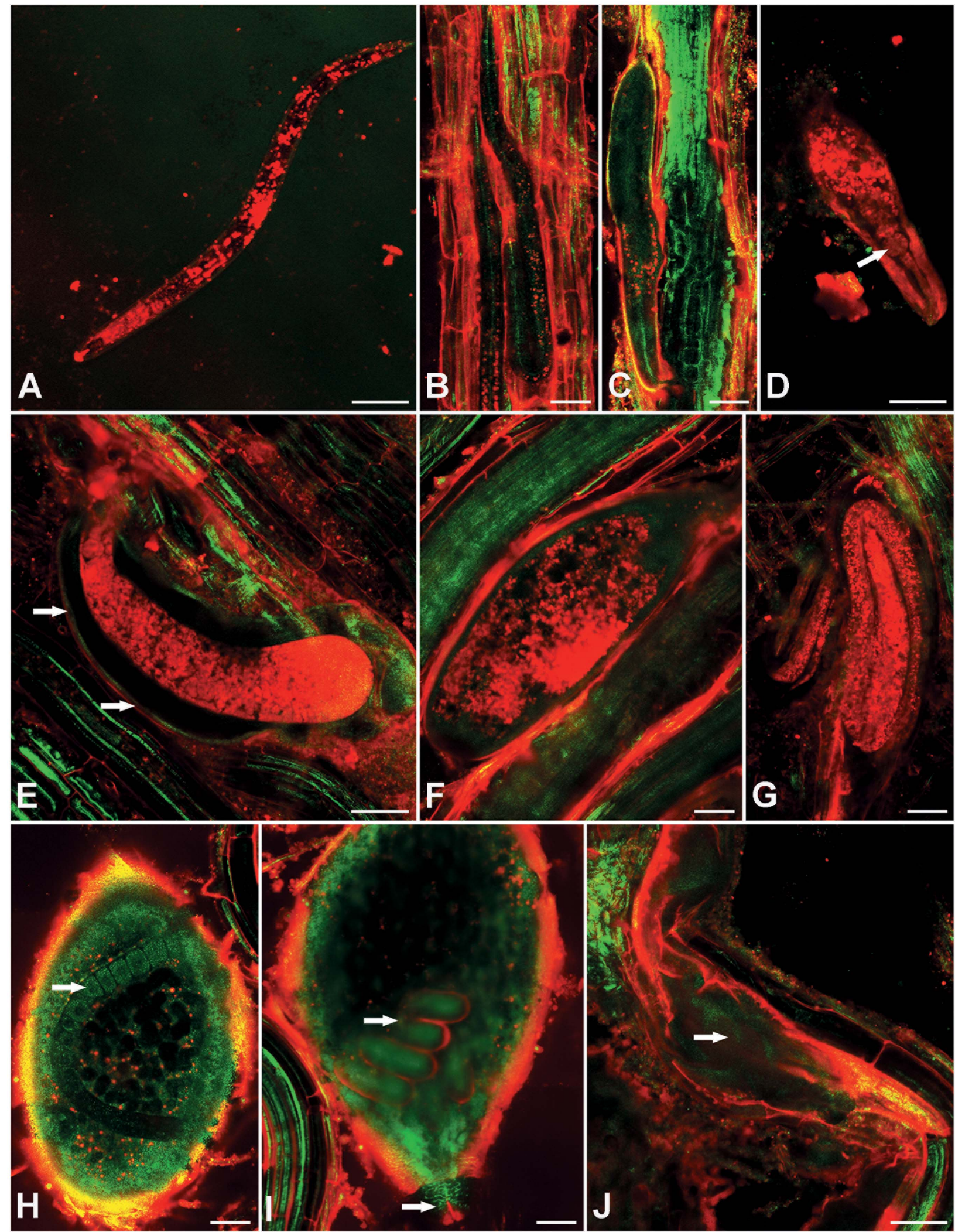

Fig. 3. Heterodera schachtii stained with $\mathrm{PKH} 26$. A, H. schachtii second-stage juveniles (J2s) 3 days after labeling with $\mathrm{PKH} 26$ outside of plant, stored at $4{ }^{\circ} \mathrm{C}$ until imaging. Intense fluorescence of intestinal lipids. All other pictures show nematodes in planta. B, Intracellular migration of J2 at 10 days after inoculation (DAI). C, J3 at 14 DAI begins to break through root cortex. D, Developing J3 or J4 with median bulb (arrow) at 22 DAI. E, Molting J3 at 23 DAI. Arrows indicate shed cuticle. F, Developing female at J4 stage, 23 DAI. G, Developing male at J4 stage, 23 DAI. H, Adult female during oogenesis (arrow indicates developing eggs) at 23 DAI. I, Mature eggs (top arrow) and vulval cone (bottom arrow) at 28 DAI. J, Syncytium (arrow) at 28 DAI. Scale bars $=50 \mu \mathrm{m}$. 
To resolve these problems, an optimized method to observe plant-nematode interactions based on fluorescently labeling nematodes with PKH26 and following behavior inside roots using plants grown in micro-ROC was developed. The technique has distinct advantages over previously used imaging methods. Most importantly, it enables nondestructive long-term observations of plant-nematode interactions over the entire life of the parasite. Furthermore, host plants are grown in potting soil and roots are
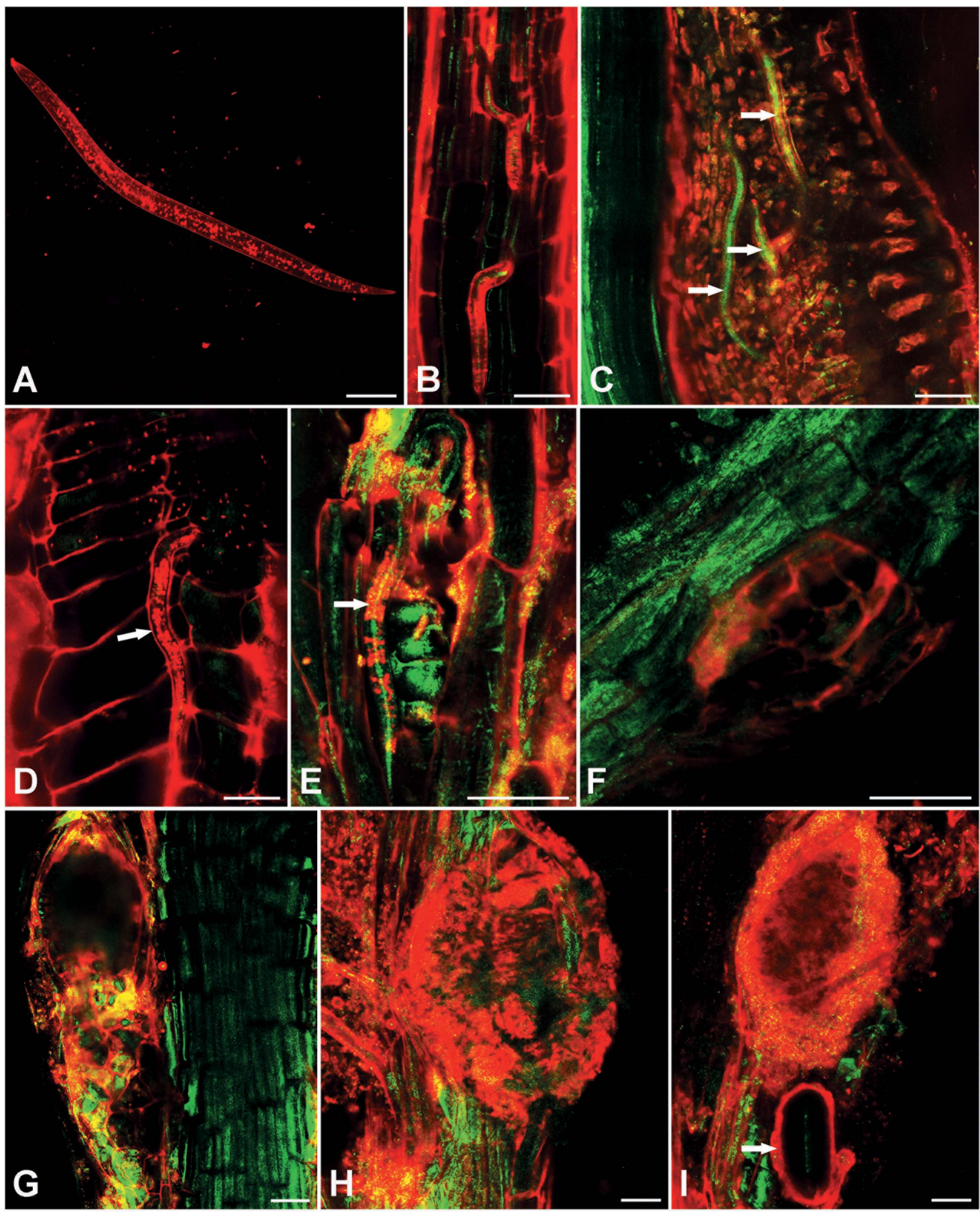

Fig. 4. Meloidogyne chitwoodi stained with PKH26. A, M. chitwoodi second-stage juveniles (J2s) 3 days after labeling with $\mathrm{PKH} 26$ outside of plant, stored at $4{ }^{\circ} \mathrm{C}$ until imaging. Intense fluorescence of intestinal lipids. All other pictures show nematodes in planta. B, Migrating J2 at 3 days after inoculation (DAI). C, Late parasitic J2 and developing gall at 12 DAI. D and E, J2 (arrow) at 12 DAI during intercellular migration. F, Early giant cell formation at 12 DAI. G, J3 or J4 breaking through the root cortex at 15 DAI. $\mathbf{H}$ and I, Posterior end of female surrounded by gall tissue at 27 DAI with egg (arrow). Scale bars $=50 \mu \mathrm{m}$. 
not exposed to light. These growing conditions sidestep potential problems with media composition that can influence the ability of nematodes to infect (43) and avoid the need to surface-sterilize nematodes with compounds such as mercuric chloride, which is lethal at high concentrations and can have a negative impact on host-parasite interactions, even at low concentrations, by interfering with nematode neurotransmission, olfaction, and locomotion (31). Roots grown with exposure to light show a greater total
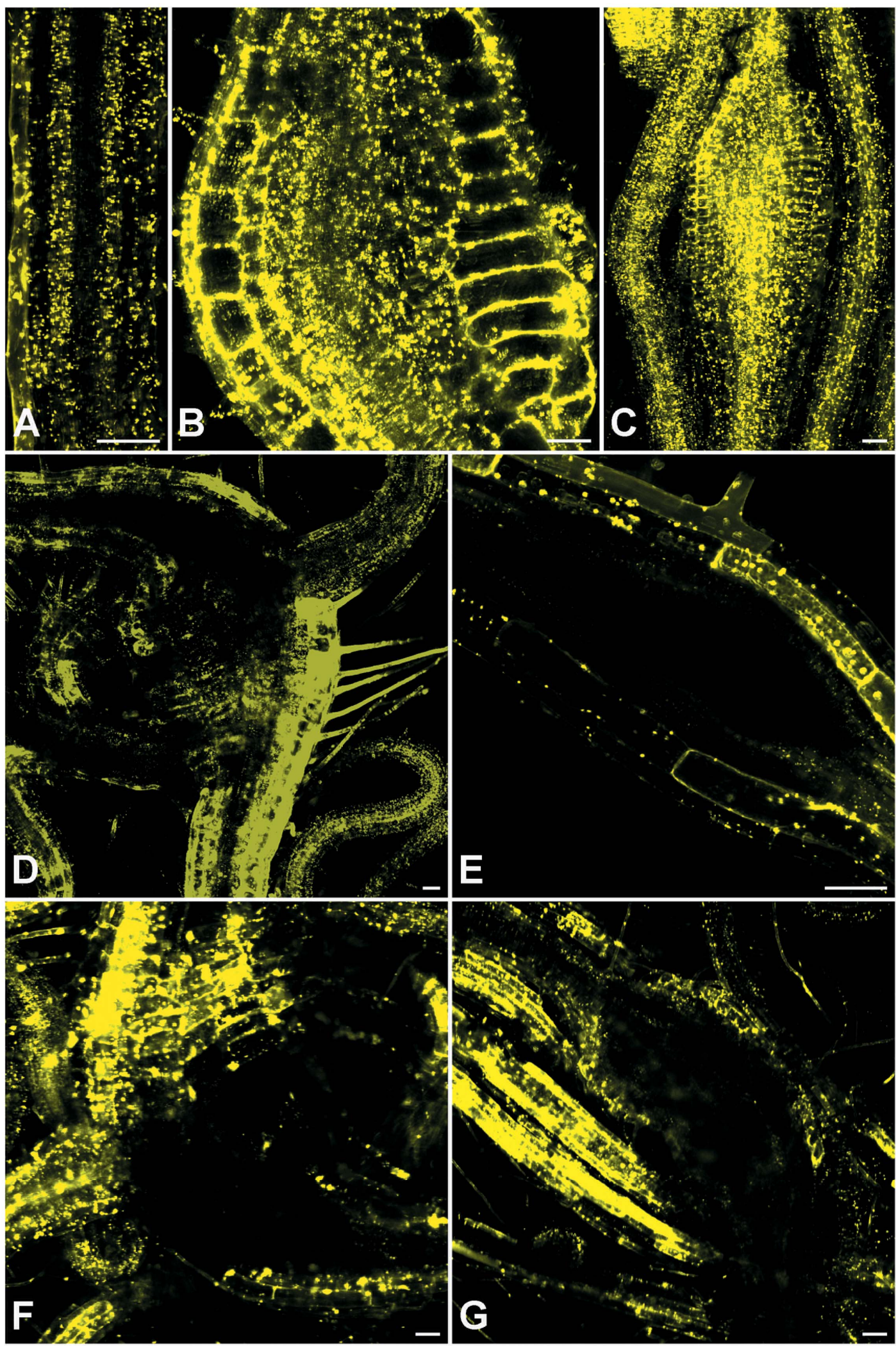

Fig. 5. Peroxisomes tagged with yellow fluorescent protein (YFP) in transgenic Arabidopsis roots infected with Meloidogyne chitwoodi. A, Distribution of YFPtagged peroxisomes in uninfected roots 22 days after germination in microscopy rhizosphere chambers. B, Early M. chitwoodi gall formation at 3 days after inoculation (DAI). C, Increased peroxisome concentration in developing gall region compared with surrounding uninfected root tissue at 8 DAI. D to G, Reduction of peroxisome concentration in developing gall region compared with surrounding uninfected root tissue at 12 DAI (D), 19 DAI (E), and 27 DAI (F and G). Scale bars $=50 \mu \mathrm{m}$. 
length, more branching, more root hairs, and bursts of reactive oxygen species $(52,53)$, traits that can influence nematode infectivity. Additionally, exposure of nematode-infected root systems to light is known to cause the formation of chloroplasts in feeding sites of sedentary endoparasites $(40,45)$. Recent studies provide evidence for molecular cross-talk between photosynthesis, plant immunity, and chloroplast-derived reactive oxygen species involved in defense responses $(13,20,55)$. Plant-nematode interaction studies based on culture conditions that permit permanent light exposure of roots grown in agar are altering the relationships between the nematode and its host, thereby possibly inviting systematic errors and confounding variables that can easily be avoided with the technique presented here. In this study, we have limited our observations to A. thaliana due to its ease of culture but micro-ROC have been used to study root biology in a wide variety of plants, including tobacco, wheat, and onion (M. Knoblauch, unpublished data). The versatility of the micro-ROC growing system and the ability of PKH26 to stain a wide range of plant-parasitic nematodes suggest that this technique can be applied in diverse plant-nematode pathosystems.

PKH26 labeling was extremely stable for the duration of the life cycle of all three nematode species tested here. The dye accumulated predominantly in the intestinal lipid granules and, to a lesser degree, in the surface coat of nematodes. In our experiments, we saw no obvious change in the behavior and biology of the nematodes stained with PKH26 and, thus, have no reason to believe that other aspects of the nematodes' biology are affected by the staining. In nonfeeding $\mathrm{J} 2 \mathrm{~s}$ of $H$. schachtii and $M$. chitwoodi, the intensity of $\mathrm{PKH} 26$ fluorescence temporarily decreased but, once feeding commenced and the intestinal lipid reservoirs were replenished, fluorescence returned to high intensity in juveniles and young adults before it decreased again in adults. This pattern is consistent with the changes in lipid concentration in developing nematodes and the abundance of lipid droplets in the intestine $(1,10,25,30)$. Furthermore, lipid droplets are dynamic and can separate into smaller droplets or merge into larger ones (39), which suggests that the observed changes in PKH26 fluorescence throughout feeding and nonfeeding nematode life stages mirror this dynamic by incorporating stain from older, previously labeled lipid droplets into newly formed ones. Nematodes were labeled fluorescently almost immediately upon PKH26 exposure, which points at a diffusion mechanism across cell membranes rather than ingestion of the dye. However, PKH26 was unable to cross the nematode egg shell and did not label developing juveniles inside eggs (data not shown). Interestingly, we found that the giant cells of PKH26-labeled root-knot nematodes also displayed PKH26-specific fluorescence. Giant cells are known to develop massive ingrowths of the plasma membrane, which increase their internal surface area 10 times or more and lead to a corresponding increase in membrane lipids (23). Thus, it is feasible that the giant-cell-specific fluorescence observed here results from incorporation of $\mathrm{PKH} 26$ that was released into the feeding site by the nematode. In control plants that were infected with nonlabeled nematodes, no comparable fluorescence could be observed.

To validate the use of micro-ROC to image nematode-induced changes in plant cells during the infection process, the response of peroxisomes to root-knot nematode infection was studied. We found that the number of plant peroxisomes strongly increased in early giant cells as early as 3 DAI compared with uninfected cells. At later stages of the infection process, peroxisomes were significantly less abundant in giant cells than in uninfected plant cells. Peroxisomes are found in all eukaryotes and are involved in both reactive oxygen species decomposition and production (8). Hydrogen peroxide, a universal cell stress signal, can induce the formation of new peroxisomes in plants and animals (29). The changes in peroxisome abundance in root-knot nematode-infected plant cells observed in this study mirror changes in the transcript level of peroxisome-specific genes in infected plants. Using differential display assays, Vercauteren and co-authors (46) showed that the Arabidopsis peroxidase gene ATP6a was induced in young giant cells and endodermal cells at 3 to 7 DAI but was restricted to endodermal cells at later time points between 14 and 21 DAI, which corresponds to the location of peroxisomes observed here. Similarly, Arabidopsis peroxidase and catalase genes were found to be downregulated in giant cells in large-scale microarray experiments $(3,21)$. We hypothesize that root-knot nematodes induce, directly or indirectly, a degradation of peroxisomes in developing giant cells to protect their feeding sites from peroxisome-derived reactive oxygen species involved in plant defense responses.

In summary, the combined use of micro-ROC and fluorescent labels has the potential to significantly advance fundamental understanding of plant-nematode interactions. Further, it has broad appeal for studying rhizosphere biology in general when applied to other plant-associated organisms such as bacteria, oomycetes, or fungi.

\section{ACKNOWLEDGMENTS}

This research was supported by funding from the Washington State Department of Agriculture, Washington State Potato Commission, Idaho Potato Commission, and Washington Grain Commission to A.A.E. PPNS Number 0622, Department of Plant Pathology, College of Agricultural, Human, and Natural Resource Sciences, Agricultural Research Center, Project Number WNP00744, Washington State University, Pullman 99164-
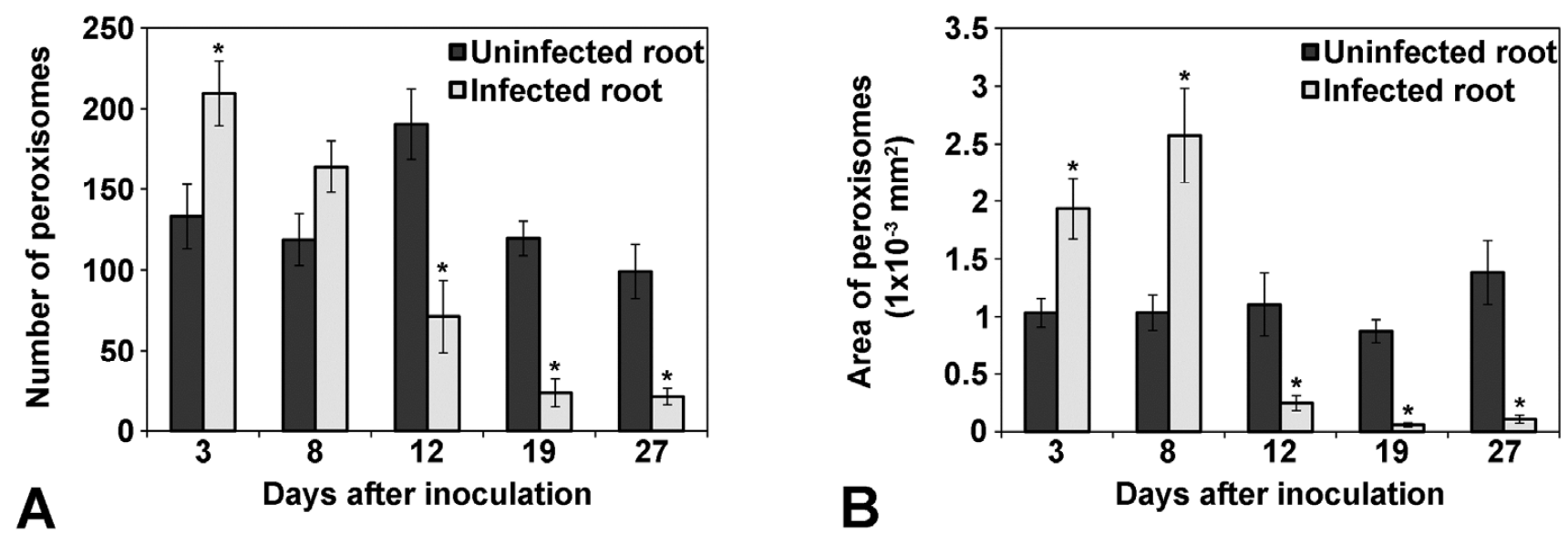

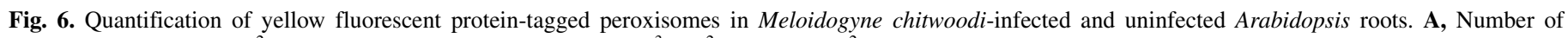
peroxisomes per $0.01 \mathrm{~mm}^{2}$ of root. B, Area of peroxisomes $\left(1 \times 10^{-3} \mathrm{~mm}^{2}\right)$ per $0.01 \mathrm{~mm}^{2}$ of root. 
6430. We thank C. Davitt and V. Lynch-Holm (Franceschi Microscopy \& Imaging Center, Washington State University) for excellent technical assistance with confocal microscopy.

\section{LITERATURE CITED}

1. Atkinson, H. J., and Harris, P. D. 1988. Changes in nematode antigens recognized by monoclonal antibodies during early infections of soya beans with the cyst nematode Heterodera glycines. Parasitology 98:479-487.

2. Banora, M. Y., Rodiuc, N., Baldacci-Cresp, F., Smertenko, A., BleveZacheo, T., Mellilo, M. T., Karimi, M., Hilson, P., Evrand, J. L., Favery, B., Engler, G., Abad, P., and de Almeida-Engler, J. 2011. Feeding cells induced by phytoparasitic nematodes require $\gamma$-tubulin ring complex for microtubule reorganization. PLoS Pathog. 7:e1002343.

3. Barcala, M., García, A., Cabrera, J., Casson, S., Lindsey, K., Favery, B., García-Casado, G., Solano, R., Fenoll, C., and Escobar, C. 2010. Early transcriptomic events in microdissected Arabidopsis nematode-induced giant cells. Plant J. 61:698-712.

4. Bird, A. F. 1979. A method of distinguishing between living and dead nematodes by enzymatically induced fluorescence. J. Nematol. 11:103105.

5. Blair, L., Perry, R. N., Oparka, K., and Jones, J. T. 1999. Activation of transcription during the hatching process of the potato cyst nematode Globodera rostochiensis. Nematology 1:103-111.

6. Böckenhoff, A., Prior, D. A. M., Grundler, F. M. W., and Oparka, K. J. 1996. Induction of phloem unloading in Arabidopsis thaliana roots by the parasitic nematode Heterodera schachtii. Plant Physiol. 112:1421-1427.

7. Clough, S. J., and Bent, A. F. 1998. Floral dip: a simplified method for Agrobacterium-mediated transformation of Arabidopsis thaliana. Plant $\mathrm{J}$. 16:735-743.

8. del Río, L. A., Corpas, F. J., Sandalio, L. M., Palma, J. M., Gómez, M., and Barroso, J. B. 2002. Reactive oxygen species, antioxidant systems and nitric oxide in peroxisomes. J. Exp. Bot. 53:1255-1272.

9. Desai, M., and Hu, J. 2008. Light induces peroxisome proliferation in Arabidopsis seedlings through the photoreceptor phytochrome $\mathrm{A}$, the transcription factor HY5 HOMOLOG, and the peroxisomal protein PEROXIN11b. Plant Physiol. 146:1117-1127.

10. Dropkin, V. H., and Acedo, J. 1974. An electron microscopic study of glycogen and lipid in female Meloidogyne incognita (root-knot nematode). J. Parasitol. 60:1013-1021.

11. Elling, A. A. 2013. Major emerging problems with minor Meloidogyne species. Phytopathology 103:1092-1102.

12. Froehlich, D. R., Mullendore, D. L., Jensen, K. H., Ross-Elliott, T. J., Anstead, J. A., Thompson, G. A., Pélissier, H. C., and Knoblauch, M. 2011. Phloem ultrastructure and pressure flow: sieve-element-occlusionrelated agglomerations do not affect translocation. Plant Cell 23:44284445.

13. Göhre, V., Jones, A. M. E., Sklenár, J., Robatzek, S., and Weber, A. P. M. 2012. Molecular crosstalk between PAMP-triggered immunity and photosynthesis. Mol. Plant-Microbe Interact. 25:1083-1092.

14. Golinowski, W., Grundler, F. M. W., and Sobczak, M. 1996. Changes in the structure of Arabidopsis thaliana during female development of the plant-parasitic nematode Heterodera schachtii. Protoplasma 194:103-116.

15. Goto, D. B., Fosu-Nyarko, J., Sakuma F., Sadler, J, Flottman-Reid, M., Uehara, T., Kondo, N., Yamaguchi, J., and Jones, M. G. K. 2010. In planta observation of live fluorescent plant endoparasitic nematodes during early stages of infection. Nematol. Res. 40:15-19.

16. Guhl, K. E., Czymmek, K., and Sherrier, D. J. 2004. Multi-photon microscopy as a tool to track nematode infection. Microsc. Microanal. 10 (Suppl.):214-215.

17. Humphreys-Pereira, D. A., and Elling, A. A. 2014. Morphological analysis of males and second-stage juveniles of Meloidogyne chitwoodi. Nematology 16:149-162.

18. Hussey, R. S., and Barker, K. R. 1973. A comparison of methods of collecting inocula of Meloidogyne spp., including a new technique. Plant Dis. Rep. 57:1025-1028.

19. Hussey, R. S., and Mims, C. W. 1991. Ultrastructure of feeding tubes formed in giant-cells induced in plants by the root-knot nematode Meloidogyne incognita. Protoplasma 162:99-107.

20. Islam, S. Z., Baradoost, M., Bekal, S., and Lambert, K. 2008. Red lightinduced systemic disease resistance against root-knot nematode Meloidogyne javanica and Pseudomonas syringae pv. tomato DC 3000. J. Phytopathol. 156:708-714.

21. Jammes, F., Lecomte, P., de Almeida-Engler, J., Bitton, F., MartinMagniette, M. L., Renou, J. P., Abad, P., and Favery, B. 2005. Genomewide expression profiling of the host response to root-knot nematode infection in Arabidopsis. Plant J. 44:447-458.

22. Jones, J. T., Haegeman, A., Danchin, E. G. J., Gaur, H. S., Helder, J., Jones, M. G. K., Kikuchi, T., Manzanilla-López, R., Palomares-Rius, J.
E., Wesemael, W. M. L., and Perry, R. N. 2013. Top 10 plant-parasitic nematodes in molecular plant pathology. Mol. Plant Pathol. 14:946-961.

23. Jones, M. G. K., and Dropkin, V. H. 1976. Scanning electron microscopy of nematode-induced giant transfer cells. Cytobios 15:149-161.

24. Jones, M. G. K., and Northcote, D. H. 1972. Multinucleate transfer cells induced in Coleus roots by the root-knot nematode, Meloidogyne arenaria. Protoplasma 75:381-395.

25. Krusberg, L. R., Hussey, R. S., and Fletcher, C. L. 1973. Lipid and fatty acid composition of females and eggs of Meloidogyne incognita and M. arenaria. Comp. Biochem. Physiol. 45:335-341.

26. Kyndt, T., Vieira, P., Gheysen, G., and de Almeida-Engler, J. 2013. Nematode feeding sites: unique organs in plant roots. Planta 238:807-818.

27. Linford, M. B. 1940. A miniature root-observation box. Phytopathology 30:348-349.

28. Linford, M. B. 1942. The transient feeding of root-knot nematode larvae. Phytopathology 32:580-589.

29. Lopez-Huertas, E., Charlton, W. L., Johnson, B., Graham, I. A., and Baker, A. 2000. Stress induces peroxisome biogenesis genes. EMBO J. 19:6770-6777.

30. Marwah, R., and Khera, S. 1985. Histochemical localization of proteins, carbohydrates and lipids in females of Meloidogyne incognita. Riv. Parasitol. 46:313-322.

31. McElwee, M. K., and Freedman, J. H. 2011. Comparative toxicology of mercurials in Caenorhabditis elegans. Environ. Toxicol. Chem. 30:21352141.

32. Mendoza, M. E. L. D., Modha, J., Roberts, M. C., Curtis, R., and Kusel, J. R. 2000. Changes in the lipophilicity of the surfaces of Meloidogyne incognita and Haemonchus contortus during exposure to host signals. Parasitology 120:203-209.

33. Mitchum, M. G., Hussey, R. S., Baum, T. J., Wang, X., Elling, A. A., Wubben, M., and Davis, E. L. 2013. Nematode effector proteins: an emerging paradigm of parasitism. New Phytol. 199:879-894.

34. Modha, J., Kusel, J. R., and Kennedy, M. W. 1995. A role for second messengers in the control of activation-associated modification of the surface of Trichinella spiralis infective larvae. Mol. Biochem. Parasitol. 72:141-148.

35. Modha, J., Piedrafita, D., Roberts, M. C., Kusel, J. R., and Kennedy, M. W. 2000. Secretion of IL-12 by murine macrophages activated by immunoglobulin receptor-mediated internalization of the surface coat of Trichinella spiralis larvae. Parasite Immunol. 22:115-120.

36. Nelson, B. K., Cai, X., and Nebenführ, A. 2007. A multicolored set of in vivo organelle markers for co-localization studies in Arabidopsis and other plants. Plant J. 51:1126-1136.

37. Nickle, W. R., ed. 1991. Manual of Agricultural Nematology. Marcel Dekker Inc., New York.

38. Nobre, M. J. G., von Mende, N., Dolan, L., Schmidt, K. P., Evans, K., and Mulligan, B. 1995. Immunolabeling of cell surfaces of Arabidopsis thaliana roots following infection by Meloidogyne incognita. J. Exp. Bot. 46:1711-1720

39. Olofsson, S. O., Boström, P., Lagerstedt, J., Andersson, L., Adiels, M., Perman, J. Rutberg, M., Li, L., and Borén, J. 2009. The lipid droplet: A dynamic organelle, not only involved in the storage and turnover of lipids. Pages 1-26 in: Cellular Lipid Metabolism. C. Ehnholm, ed. SpringerVerlag, Berlin.

40. Orion, D., and Wergin, W. P. 1982. Chloroplast differentiation in tomato root galls induced by the root-knot nematode Meloidogyne incognita. J. Nematol. 14:77-83.

41. Pece, S., Tosoni, D., Confalonieri, S., Mazzarol, G., Vecchi, M., Ronzoni, S., Bernard, L., Viale, G., Pelicci, P. G., and Di Fiore, P. P. 2010. Biological and molecular heterogeneity of breast cancers correlates with their cancer stem cell content. Cell 140:62-73.

42. Schroeder, N. E., and MacGuidwin, A. E. 2007. Incorporation of a fluorescent compound by live Heterodera glycines. J. Nematol. 39:43-49.

43. Sijmons, P. C., Grundler, F. M. W., von Mende, N., Burrows, P. R., and Wyss, U. 1991. Arabidopsis thaliana as a new model host for plantparasitic nematodes. Plant J. 1:245-254.

44. Sobczak, M., Golinowski, W., and Grundler, F. M. W. 1997. Changes in the structure of Arabidopsis thaliana roots induced during development of males of the plant parasitic nematode Heterodera schachtii. Eur. J. Plant Pathol. 103:113-124.

45. Urwin, P. E., Møller, S. G., Lilley, C. J., McPherson, M. J., and Atkinson, H. J. 1997. Continual green-fluorescent protein monitoring of cauliflower mosaic virus $35 \mathrm{~S}$ promoter activity in nematode-induced feeding cells in Arabidopsis thaliana. Mol. Plant-Microbe Interact. 10:394-400.

46. Vercauteren, I., Van Der Schueren, E., Van Montagu, M., and Gheysen, G. 2001. Arabidopsis thaliana genes expressed in the early compatible interaction with root-knot nematodes. Mol. Plant-Microbe Interact. 14:288-299.

47. Vieira, P., Banora, M. Y., Castagnone-Sereno, P., Rosso, M. N., Engler, G., and de Almeida-Engler, J. 2012. An immunocytochemical procedure 
for protein localization in various nematode life stages combined with plant tissues using methylacrylate-embedded specimens. Phytopathology 102:990-996

48. Vieira, P., Danchin, E. G. J., Neveu, C., Crozat, C., Jaubert, S., Hussey, R. S., Engler, G., Abad, P., de Almeida-Engler, J., Castagnone-Sereno, P., and Rosso, M. N. 2011. The plant apoplasm is an important recipient compartment for nematode secreted proteins. J. Exp. Bot. 62:1241-1253.

49. Vieira, P., Engler, G., and de Almeida-Engler, J. 2012. Whole-mount confocal imaging of nuclei in giant feeding cells induced by root-knot nematodes in Arabidopsis. New Phytol. 195:488-496.

50. Wyss, U., and Grundler, F. M. W. 1992. Feeding behavior of sedentary plant parasitic nematodes. Neth. J. Plant Pathol. 98 (Suppl.):165-173.

51. Wyss, U., Grundler, F. M. W., and Münch, A. 1992. The parasitic behaviour of second-stage juveniles of Meloidogyne incognita in roots of Arabidopsis thaliana. Nematologica 38:98-111.
52. Xu, W., Ding, G., Yokawa, K., Baluska, F., Li, Q. F., Liu, Y., Shi, W., Liang, J., and Zhang, J. 2013. An improved agar-plate method for studying root growth and response of Arabidopsis thaliana. Sci. Rep. 3:1273.

53. Yokawa, K., Kagenishi, T., Kawano, T., Mancuso, S., and Baluska, F. 2011. Illumination of Arabidopsis roots induces immediate bursts of ROS production. Plant Signal. Behav. 6:1460-1464.

54. Zunke, U. 1990. Observations on the invasion and endoparasitic behavior of the root lesion nematode Pratylenchus penetrans. J. Nematol. 22:309320.

55. Zurbriggen, M. D., Carrillo, N., Tognetti, V. B., Melzer, M., Peisker, M., Hause, B., and Hajirezaei, M. R. 2009. Chloroplast-generated reactive oxygen species play a major role in localized cell death during the nonhost interaction between tobacco and Xanthomonas campestris pv. vesicatoria. Plant J. 60:962-973. 